\title{
A Close Look at the Physical Fitness Levels of Elementary Age Students with Intellectual Disabilities
}

\author{
Kathryn L. DAVIS • Guili ZHANG • Patricia S. HODSON \\ Boni B. BOSWELL・ James T. DECKER
}

\begin{abstract}
Tntroduction: Little is known about the physical fitness levels of 1 school-aged children with intellectual disabilities, when comparing groups of healthy weight and unhealthy weight. Purpose: To compare the fitness levels of healthy weight and unhealthy weight children with intellectual disabilities in three elementary schools in the southeastern United States. Methods: Four fitness measures (PACER, Modified Curl-Ups, Modified Pullups, and the Back-Saver Sit-and-Reach) were obtained from 26 youth (10 girls, 16 boys; $10.01+1.22)$. Additionally, fitness levels of students who were overweight/obese were statistically compared with those with healthy weights. Results: A pattern of overall low fitness levels was found in comparison to criterion-referenced standards regarding all of the fitness measures, and students who were overweight/obese performed poorly compared to those with healthy weight. The only statistically significant finding was the comparison of healthy and unhealthy weight groups on the modified pullup. Conclusions: The study suggests a strong need for adaptive physical education and active recess programs that are appropriate for developing the fitness levels of elementary students with intellectual disabilities.
\end{abstract}

Keywords: intellectual disabilities, adaptive physical education.

\section{Introduction}

The need to improve physical fitness in school-aged children in the United States has received considerable attention ever since Kraus and Hirschland (1954) indicated American children were less fit than European children. In recent years, concern for the health-related physical fitness of all children in American schools has increased because of the current childhood obesity epidemic. However, much less attention has been specifically focused on the 
measurement and assessment of health-related physical fitness among children with disabilities (Winnick, 2005b). The benefits of physical fitness are universal for all children and can potentially promote inclusion, minimize deconditioning, optimize physical functioning, and enhance overall well-being (Murphy \& Carbone, 2008). Children with disabilities are especially at risk for poor health, as a result of sedentary lifestyles (Ayvazoglu, Ratliffe, \& Kozub, 2004).

In 2006, the US Department of Education began using the designation "intellectually disabled" (ID) for individuals historically categorized as "mentally retarded." The term ID has evolved to include the same population of individuals previously diagnosed with mental retardation in definition and classification (AAMR, 2002). A few examples of syndromes that are associated with ID include: attention-deficit/hyperactivity disorder (ADHD), autistic syndrome disorder, and Down syndrome (Pitetti, Beets, \& Combs, 2009). Children with ID are one of the largest populations of students with disabilities in school settings (Faison-Hodge \& Poretta, 2004). These children with ID are likely to be more restricted in their participation, have lower levels of fitness, and have higher levels of obesity than their peers without disabilities (Frey \& Chow, 2006). Specifically, it has been consistently reported that youth with ID have lower levels of cardiovascular endurance and muscular strength, as well as higher body mass index measures, in comparison to their typically developing peers (Pitetti \& Yarmer, 2002; Pitetti, Yarmer, \& Fernhall, 2001).

Because of the lower levels of fitness found in children with ID, it was assumed that they also had lower physical activity patterns than their nondisabled peers. However, to assume that there is a direct relationship between physical fitness and physical activity is unsettling, because $10-20 \%$ of the variance in physical fitness can be accounted for by physical activity (Pitetti, Beets, \& Combs, 2009). Therefore, physical activity patterns and physical fitness measures cannot be viewed as equal indicators of health. However, healthrelated fitness components are affected by habitual physical activity, and physical activity provides a conditioning benefit for health-related fitness components (Winnick, 2005a). As Winnick stated, "clearly health, health-related physical fitness, and physical activity affect and are affected by each other" (2005a, p. 323). In addition, sedentary behavior in children with intellectual disabilities (ID) may be influenced by insufficient motor or physical fitness, because competence in movement is crucial to activity participation (Okely, Booth, \& Chey, 2004). Because having higher levels of physical fitness is important to the functional health needed for everyday living and for the prevention of disease and obesity, attempts should be made to evaluate the physical fitness of children with ID. 
Another compounding factor in studying physical fitness in children with ID is that children with disabilities may not receive adequate levels of PA in order to meet the Surgeon General's recommendations for "moderate to vigorous physical activity" (MVPA). The 1996 Surgeon General's Report on physical activity and health highlights the need to identify and track physical activity patterns of children with ID (Faison-Hodge \& Poretta, 2004). Research on physical activity and children with disabilities has been identified as a significant public health priority (Temple, Frey, \& Stanish, 2006).

There have been numerous efforts to study the rise in obesity among typically developing children, but it is unclear if these studies can be generalized to children with ID because of the documentation that children with ID experience difficulties with motor ability and they exhibit low physical fitness levels (Frey \& Chow, 2006). There are indications that a high body mass index (BMI) negatively affects motor performance and physical fitness in typically developing children (Okely, Booth, \& Chey, 2004), but attempts to link high BMI with physical fitness for children with ID have yielded mixed results (Frey \& Chow, 2006). Youth with ID perform poorly on motor and fitness assessments (Eichstaedt \& Lavay, 1992), but it is unclear if there is a direct relationship between overweight/ obesity and the five components of physical fitness. Therefore, there is a need to better understand the factors that contribute to the health and well-being children with ID.

The primary goals of this study were to assess the physical fitness levels of elementary-aged students with ID, and to determine if there was a relationship between fitness levels and BMI in this population. This study sought to answer the following two questions: (a) What are the current fitness levels of a selected group of children with ID, aged 8-12, and (b) What is the relationship between physical fitness and BMI in this population?

\section{Methodology}

Participants. A total of twenty-six elementary school children (10 girls, 16 boys; $10.01+1.22$ ) with mild to moderate ID from three Southeastern elementary schools participated in the project. Twelve of the 26 students were AfricanAmerican, 12 were Caucasian, and two were Hispanic. The 26 participants in this study were representative of what one would find in any self-contained placement class. All participants had been classified as having mild to moderate ID by school district personnel. Although IQ scores were not available, verification that the sample represented youth with mild ID was based on strong adherence to school placement according to IQ and teacher confirmation. In this school district, the majority of youth with ID are placed in segregated self-contained classes 
according to learning ability and diagnosis. Informed parental consent, along with district and school administration approval, was obtained before participation in the study. The study was approved by the University Institutional Review Board.

The three elementary schools were randomly selected from the seven elementary schools in the district that included self-contained special education classes. Participants were from three different elementary schools $(K-5)$ of approximately 600 students each. The sample design was purposive in that the participants had to meet the criterion for mild to moderate ID, they must be enrolled in a self-contained classroom, and they could not have any limiting physical condition that would hinder their ability to engage in routine PA (Pitetti, Beets, \& Combs, 2009). The three special-needs classes contained 5-10 students, a licensed special education teacher who was assisted by two paraeducators. The amount of support (i.e., paraeducators) for each child depended on the needs of the child as outlined by their individualized educational plan. A total of 38 students with mild to moderate ID were enrolled in the three special needs classes, and were invited to participate in this study. Only 31 consented to participate, and five of those were unable to participate or complete all measurements due to absence or co-concurring disabilities. These co-concurring disabilities impacted their ability to participate in the fitness testing (e.g. sensory sensitivity associated with autism, tests not validated for those with physical disabilities) because their data could lead to unreliable results (Frey \& Chow, 2006).

In all three settings, the self-contained classes received physical education from a licensed physical educator twice per week, with at least two paraeducators assisting. During class instructional activities in physical education (45 minute periods), the curriculum involved games and physical activities that were focused on continuous movement and reinforcement of concepts learned in the selfcontained classes. Thirty minutes of active recess was required by the state for all children $\mathrm{K}-8$, but these three self-contained classes did not receive any structured recess. During recess, the special education teachers and paraeducators would observe for safety, but they did not direct or suggest activity.

Data Collection. Data collection was conducted during a one-week period during the last month of the school year. The physical educator at each school, along with the special education teachers and their assistants, assisted the research team with data collection at each school. The research team consisted of five senior researchers and two graduate students, who were trained in the assessment protocols. Between these researchers, they had over 100 years of experience working with children with disabilities and conducting assessments in schools and in clinical field settings. The BMI classifications of "healthy weight" and "unhealthy weight" (overweight/obese) were determined by using 
the FitnessGram criterion-referenced standards, called "Healthy Zones." The FitnessGram testing protocol is currently endorsed by the American Alliance for Health, Physical Education, Recreation, and Dance (Meredith \& Welk, 2007), and FitnessGram was used as an important reference in developing the Brockport Physical Fitness Test (BPFT) for students with disabilities (Winnick, 2005b). These "Healthy Zone" standards are also supported by the FitnessGram Scientific Advisory Board. These "Healthy Zone" standards were used for the BMI classifications because there is no consensus for a single set of standards to be used for students with intellectual disabilities.

While there are numerous tests and test batteries to measure the five components of physical fitness for typically developing children, there is only one test battery that has specifically been designed for the special needs of children with disabilities (Winnick, 2005a). The fitness measures were selected from the BPFT because this test battery is valid and reliable for students with ID (Winnick \& Short, 1999). The five testing items used in this study were selected using BPFT guidelines, and they were administered with the careful use of verbal prompting (there were no participants with hearing impairments) and appropriate motivation techniques for students with ID. These measures were chosen because of their roles as important indicators of health-related fitness. The measures included: (a) body mass index (BMI), (b) the 16-meter modified Progressive Aerobic Cardiovascular Endurance Run (PACER) to measure cardiovascular endurance, (c) the modified curl-up test of abdominal strength and endurance, (d) the modified pull-up test to measure arm strength, and (e) the back-saver sit-and-reach (BSSR) test to measure flexibility. Testing was conducted in a gymnasium in two schools, and in an indoor multi-purpose room in one school.

Body Mass Index (BMI). Body Mass Index (BMI) provides an indication of the appropriateness of a child's weight relative to height. BMI is usually calculated from height and weight data and is derived from the equation:

$$
\mathrm{BMI}=\text { body weight }(\mathrm{kg}) / \text { height } 2(\mathrm{~m}) .
$$

Estimates of obesity level based on height and weight (BMI) result in an acceptable level of 5\% to 6\% error because body weight reflects muscle and bone mass as well as fat mass (Lohman, 1981). Standing height (cm) was measured without shoes to the nearest $0.1 \mathrm{~cm}$ using a portable stadiometer (Model 214, range: 20-200 cm; Seca, Hamburg, Germany). Body weight (kg) was measured to the nearest $0.1 \mathrm{~kg}$ using a digital weight scale (Model DG-66, maximum: 150 kg; Seca, Hamburg, Germany). Participants' ages were calculated by subtracting their date of birth from the date of assessment, using the Weill Medical College 
of Cornell University age calculator for pediatric medicine (Pon, 2009). Agespecific BMI values were calculated using the Centers for Disease Control and Prevention's BMI Calculator for Child and Teen (National Center for Health Statistics, 2010). Winnick and Short (2005b) reported sufficient concurrent and predictive validity for BMI tests in the BPFT. 'They also reported that there is sufficient reliability for the measure of BMI in the BPFT, "due to the "objective nature of the measurements that comprise BMI" (p. 368). "The reliability of $\mathrm{BMI}$ is very high because the measurement of height and weight is very precise when following a standardized protocol" (Lohman, 1994, p. 59).

PACER. Research clearly indicates that acceptable aerobic capacity are associated with a reduced risk of high blood pressure, coronary heart disease, obesity, diabetes, some forms of cancer, and other health problems (Meredith \& Welk, 2007). The 16-meter PACER test is a multistage aerobic capacity fitness test adapted from the 20-meter shuttle run test, which was found to be advantageous for youngsters with ID (Winnick \& Short, 2005a). For this study, 3-4 students with ID were tested during each round of PACER testing, and each student had an adult "partner" who verbally motivated him/her to continue running as long as possible. Cones were set at a starting line, and at a distance of 16 meters from the starting line for each student. One lap was counted for every 16-meter distance covered. The test was concluded when the participant could no longer complete a 16-meter lap. According to Meredith and Welk (2007), the PACER has demonstrated reliability and validity measured against maximal oxygen uptake (VO2 max), which is generally considered the best measure of aerobic capacity. Similarly, very high test-retest reliability for the 16-m PACER (alpha coefficients of .96 and .98) was found by Winnick and Short (2005a).

Modified Curl-Up. The modified curl-up is used to measure the abdominal strength and endurance. The modified curl-up was performed with the participants' knees flexed and their feet unanchored. The students with ID were instructed to place their hands on the top of their thighs (near their hips), and slide their hands up to the top of the knees. An adult "partner" served as a verbal motivator and counter for each student. This test was selected for use in this study because of its high reliability, validity, and adaptability (Winnick \& Short, 2005d). The intraclass coefficients (R) for the modified curl-up range from .93 to .97 (Robertson \& Magnusdottir, 1987). Effort was made in the development of the BPFT to establish an association with the FITNESSGRAM modified curl-up so that test users could switch back and forth between the two modified curl-up tests as necessary (Winnick \& Short, 2005d).

Modified Pull-Up. The modified pull-up test has the advantage of producing few zero scores, and it can result in a wide range of scores. Each participant 
in this study was positioned on his/her back with the shoulders between the uprights and directly below a bar that was set 1-2 inches beyond the child's reach. An elastic band was placed 7-8 inches below the bar. The participant started in a down position ("straight as a board") with an overhand grip. Each participant completed as many pull-ups as possible while keeping the hips and knees straight (Pate, Ross, Baumgartner, \& Sparks, 1987). A successful pull-up was completed when the child's chin was raised above the elastic band. The modified pull-up has reported reliability coefficients of .80-.90 in the studies reviewed by Plowman and Corbin (1994). Effort was made in the development of the BPFT to establish an association with the FITNESSGRAM modified pull-up so that test users could switch back and forth between the two modified pull-up tests as necessary (Winnick \& Short, 2005d).

Back-Saver Sit-and-Reach (BSSR). Flexibility and range of motion are measures that describe attributes of motion within the body. The BSSR was included in the BPFT battery in response to the health-related concern of low back pain, or the risk of children with ID developing low back pain in the future (Winnick \& Short, 2005c). The BSSR is very similar to the traditional sit-and-reach test, except that the measurement is performed on one side at a time. In this study, BSSR was tested three times on the left side, and three times on the right side. The highest score on each side was used in the data analysis because it is likely to best represent the child's true flexibility and range of motion. The advantage of the BSSR is that by testing one leg at a time, any asymmetry in hamstring flexibility can be identified with acceptable accuracy (Winnick \& Short, 1999). An intraclass reliability of .99 was reported for the BSSR (Patterson, Wiksten, Ray, Flanders, \& Sanphy, 1996).

Data Analysis. Student data were compared to the standards set forth by the FitnessGram (Meredith \& Welk, 2007) and the BPFT standards for children with disabilities (Winnick \& Short, 1999). Two standards were used to judge participants because there is no consensus for a single standard for students with ID. The FitnessGram has criterion-referenced standards, called "Healthy Zones," that are used to determine students' fitness levels based on what is optimal for good health. The FitnessGram test assesses the fitness levels of children in grades $\mathrm{K}-12$, and the raw scores are compared to the Healthy Zone scores. The Fitness Gram standards were based on expert opinion derived in part from an analysis of normative data collected in the United States and Canada (Plowman \& Corbin, 1994). The standards of the BPFT were adapted from the FitnessGram, in which standards for youngsters with ID were derived by lowering the minimal general standards by a percentage range of $25-50 \%$ (Winnick \& Short, 2005d). 
In order to investigate whether these observed differences were statistically significant, a Mann-Whitney Test (Wilcoxon, 1945; Mann \& Whitney, 1947) was conducted to compare students with unhealthy weight and those with healthy weight regarding their performance on each of the five fitness measures. The Mann-Whitney Test was used instead of the commonly known $t$ test, because the Mann-Whitney Test is a non-parametric test that is appropriate when the sample size is small and the shape of the underlying data distribution is unclear. The Mann-Whitney Test is one of the best-known non-parametric significance tests and it is used for assessing whether two samples of observations come from the same population distribution (Mann \& Whitney, 1947). The Mann-Whitney Test is virtually identical to performing an ordinary parametric two-sample $t$ test on the data after ranking over the combined samples. The significance level was set at the conventional .05 level.

\section{Results}

The performance of the participants on each of the five fitness measures were compared to the FitnessGram criterion-referenced standards and to the BPFT's age-specific and gender-specific standards. The results are reported in Table 1. The percentage of participants who met the healthy standards is listed in the "satisfactory" column, and the percentage of participants who did not meet the standards is listed in the "unsatisfactory" column.

Table 1. Participants' Performance Compared to FITNESSGRAM and BPFT Standards

\begin{tabular}{|c|c|c|c|}
\hline Measures & Standards & Satisfactory $(\%)$ & Unsatisfactory ( $\%)$ \\
\hline BMI & $\begin{array}{l}\text { FITNESSGRAM } \\
\text { BPFT }\end{array}$ & $\begin{array}{l}67 \\
67\end{array}$ & $\begin{array}{l}33 \\
33\end{array}$ \\
\hline PACER & $\begin{array}{l}\text { FITNESSGRAM } \\
\text { BPFT }\end{array}$ & $\begin{array}{l}7 \\
60\end{array}$ & $\begin{array}{l}93 \\
40\end{array}$ \\
\hline Modified Curl-up & $\begin{array}{l}\text { FITNESSGRAM } \\
\text { BPFT }\end{array}$ & $\begin{array}{l}42 \\
33\end{array}$ & $\begin{array}{l}58 \\
67\end{array}$ \\
\hline Modified Pull-up & $\begin{array}{l}\text { FITNESSGRAM } \\
\text { BPFT }\end{array}$ & $\begin{array}{l}23 \\
13\end{array}$ & $\begin{array}{l}77 \\
87\end{array}$ \\
\hline BSSR & $\begin{array}{l}\text { FITNESSGRAM } \\
\text { BPFT }\end{array}$ & $\begin{array}{l}65 \\
60\end{array}$ & $\begin{array}{l}35 \\
40\end{array}$ \\
\hline
\end{tabular}


BMI. The average BMI of the 26 students was 19.95, a value that is close to the heavier end of the healthy fitness zone based on the Fitnessgram standard. When compared to the FitnessGram healthy fitness zones, roughly 19\% of the students were overweight, $7 \%$ of the students were "at risk" of being overweight, $67 \%$ had healthy weight, and $4 \%$ were underweight. Therefore, a total of $33 \%$ had unhealthy weight and $67 \%$ had healthy weight (see Table 1). The 15 students aged 10-11 (because BPFT has standards for only these ages) were assessed using the BPFT standard in addition to the FITNESSGRAM standard; a total of $33 \%$ of them had unhealthy weight, while $67 \%$ had healthy weight.

PACER. The PACER laps completed by the participants had a mean of 11.23 laps with a wide range of 1-32 laps. Five of the students completed four laps or less. Neither the FitnessGram nor the BPFT offer lap count standards for children under the age of nine. Among the 15 students whose age ensured them a standard to compare (from either FitnessGram or BPFT), only one student $(7 \%)$ met the criteria, while the rest of the 14 students $(93 \%)$ performed well below the minimum standard set forth by FitnessGram. Among the same 15 students, nine (60\%) students met the BPFT criteria, and six students $(40 \%)$ performed well below BPFT's minimum standard.

Modified Curl-Up. Among the 26 students, only 11 students (42\%) met the minimum FitnessGram standard. Among the 15 students who were assessed using the BPFT standard, only five students (33\%) met the minimum standard.

Modified Pull-Up. As indicated in Table 2, among the 26 students, only 23\% met the minimum FitnessGram standards. Among the 15 students who were assessed using the BPFT standard, only two students (13\%) met the minimum BPFT standards.

BSSR. A student was considered as having met the minimum FitnessGram and/or BPFT standards if he or she reached the standard on either the left side or right side or both. A total of 35\% did not meet the minimum FitnessGram standard for the left side, and the same percentage did not meet the minimum standard for the right side. Among the 15 students who were compared to the BPFT standards, six students $(40 \%)$ did not meet the minimum standard for the left side, and $40 \%$ did not meet the minimum BPFT standard for the right side. 
Table 2. Healthy vs. Unhealthy Weight Fitness Comparisons Using Mann-Whitney Test

\begin{tabular}{llcccc}
\hline Measures & Group & $\mathrm{N}$ & Mean & Mann-Whitney U & $\mathrm{P}$ \\
\hline \multirow{2}{*}{ PACER } & Healthy & 18 & 12.67 & 53.5 & .30 \\
\multirow{2}{*}{ Modified Curl-up } & Unhealthy & 8 & 8 & & \\
& Healthy & 18 & 12.5 & 40 & .07 \\
Mnhealthy & 8 & 5.13 & & \\
\multirow{3}{*}{ BSSR Rified Pull-up } & Healthy & 18 & 3.44 & 34 & $.02 *$ \\
& Unhealthy & 8 & .25 & & \\
\multirow{2}{*}{ BSSR Left } & Healthy & 18 & 10.31 & 52.5 & .28 \\
& Unhealthy & 8 & 8.43 & & \\
& Healthy & 18 & 9.91 & 53.5 & .30 \\
\hline
\end{tabular}

Note: The $*$ indicates statistical significance at $\alpha=.05$ level.

Fitness Comparisons: Healthy vs. Unhealthy Weight Groups. The means of the fitness measures by group reveal a clear pattern, that is, the healthy weight group did better on all of the fitness measures obtained (see Table 2). The healthy weight group completed an average of 12.7 PACER laps, while the unhealthy group completed an average of only eight laps. The healthy weight group completed an average of 12.5 modified curl-ups, while the unhealthy groups only completed 5.1. The healthy group completed an average of 3.44 modified pullups, representing a striking difference compared to the unhealthy group's average of 0.25 . The healthy group's average BSSR score was 10.3 inches on the right side and 9.9 inches on the left side, both higher than the unhealthy group's average score of 8.4 inches and 8.8 inches on the right side and left side, respectively.

The results revealed a statistically significant difference in the modified pull-ups between the two groups, where the average number of modified pullups completed by the healthy weight group and the unhealthy weight group were 3.44 and .25; the distributions in the two groups differed significantly (MannWhitney $\mathrm{U}=34, \mathrm{n} 1=18, \mathrm{n} 2=8, \mathrm{P}=0.02$ two-tailed).

\section{Discussion}

The results of the present study suggest that children with ID do not have a higher incidence of overweight/obesity than typical children. Thus, within these schools' environments, children with ID have sufficient time to be physically 
active to increase their levels of fitness, and the pattern of results suggests that healthy weight children with ID have higher fitness levels than children with ID who have unhealthy weight. However, these statistical comparisons reveal that only one comparison, the healthy weight group did better on the modified pullup than the unhealthy weight group, was of statistical significance. Therefore, the pattern of better fitness scores in all five areas can only be suggested. Unfortunately, the results of this study lack generalization to other locations. That is, schools that have inclusive (or mainstreamed) classes or different teacherto-student ratios, and less time in physical education and recess, may experience different results for physical fitness levels in children with ID. However, these findings do indicate that the incidence of overweight/obesity in children with ID may be less than has been previously reported.

How did the current fitness levels of the 26 participants compare to the FitnessGram Healthy Zone criterion-referenced standards for typical children (Meredith \& Welk, 2007), and to the Brockport Physical Fitness Test (BPFT) criterion-referenced standards for children with disabilities (Winnick \& Short, 1999)? The results of this study also revealed that the physical fitness levels of children with ID strongly indicated low fitness levels in comparison to the healthy standards set for typical students (Meredith \& Welk, 2007), as well as the healthy standards for students with disabilities (Winnick \& Short, 1999). In all of the fitness measures obtained, high percentages of students were below the recommended minimum standards. A total of $33 \%$ of the students in the study had unhealthy weight according to the FitnessGram and the BPFT standards. By the FitnessGram standard for typical students, $93 \%$ of the participants did not meet the standard for the PACER, 58\% performed below the modified curl-up standard, $77 \%$ were not able to complete the minimum number of modified pullups, and approximately 35\% did not meet the criteria for the BSSR. Similarly, by the BPFT standard for children with ID, $40 \%$ of the participants did not meet the standard for the PACER, 67\% performed below the modified curl-up standard, $87 \%$ were not able to complete the minimum number of modified pull-ups, and approximately $40 \%$ did not meet the criteria for the BSSR.

One potential limiting factor of this study was the "dropout rate" from the original pool of participants. The parents of seven students did not sign the informed consent because of their desire for their children "not to be studied" in the research project. The special education teachers conveyed the concern of these parents that their children would be labeled as "different" by participating in a research study. Even though the remaining sample was highly reflective of the overall population of students with ID in this school system, this could have negatively affected the results. Another potential limiting factor concerns the behavioral aspects of the co-concurring conditions or behaviors associated with 
some of the participants with ID. Data from five participants (whose parents consented to participation) were not included because of the difficulty of their physical participation, their absenteeism during a majority of the tests, or their refusal to be tested. Again, this additional lack of participation may have affected the results. One other potential limiting factor was the lack of one single set of fitness standards for all age levels of children with ID. Perhaps after further research on the fitness levels of all ages of children with ID, this single set of fitness standards will be possible.

Whether the findings of this study indicate the same fitness levels of children with ID in schools across the United States is problematic for several reasons. First, only one-third of schools in the United States have separate adapted physical education classes specifically for mental and physical disabilities (Lee, Burgeson, Fulton, \& Spain, 2007). Second, the likelihood of children with ID having adapted physical education or active recess in schools with a ratio of educator/para-educator/student ratio of near 3:1 would be nominal. Third, among school districts in the United States, only 38.5\% require or recommend > 30 minutes of structured recess per day, and $12.6 \%$ do not require or recommend recess at all (Lee et al., 2007). The participants in the present study were required to have 30-minute unstructured recess periods every school day. Therefore, the results of the study may not be representative of the fitness levels of children with ID in the school districts within the United States.

The findings of this study point to an alarming reality that there is a need to promote fitness programs among elementary students with ID. The first step in addressing the problem could be identifying the factors that are contributing to the low fitness levels among children with ID. Future research needs to closely and critically interrogate the structures, processes, and practices involved that may obstruct children's participation in programs that promote physical fitness in schools. The problem needs to be investigated and addressed from multiple angles, starting with some of the potentially most influential factors. Some of those probable contributing factors might be time availability, educators' awareness level, parents' involvement, children's motivation, a lack of staff development, and the availability of adaptive physical education and physical activity programs that are suitable, feasible and sustainable for children with ID. The findings from this study could also be useful as a basis for the development of school-based adapted fitness programs (or interventions) uniquely appropriate for meeting the physical needs, and the cognitive and social needs, of students with ID. 


\section{References}

American Association for Mental Retardation [AAMR]. (2002). Mental retardation: Definition, classification, and systems support (10th ed.). Washington, DC: Author.

Ayvazoglu, N. R., Ratliffe, T., \& Kozub, F. M. (2004). Encouraging lifetime physical fitness. Teaching Exceptional Children, 37(2), 16-20.

Eichstaedt, C. B., \& Lavay, B. W. (1992). Physical activity for individuals with mental retardation: Infancy through adulthood. Champaign, IL: Human Kinetics.

Faison-Hodge, J. \& Poretta, D. (2004). Physical activity levels of students with mental retardation and students without disabilities. Adapted Physical Activity Quarterly, 21, 139-152.

Frey, G. C., \& Chow, B. (2006). Relationship between BMI, physical fitness, and motor skills in youth with mild intellectual disabilities. International Journal of Obesity, 30, 861-867.

Kraus, H., \& Hirschland, R. P. (1954). Minimus muscular fitness tests in school children. Research Quarterly for Exercise and Sport, 25, 178-188.

Lee, S. M., Burgeson, C. R., Fulton, J. E., \& Spain, C. G. (2007). Physical education and physical activity: Results from the School Health Policies and Programs Study 2006. Journal of School Health, 77, 435-463.

Lohman, T. G. (1994). Body composition. In J. R. Morrow, H. B. Falls, \& H. W. Kohl (Eds.), The Prudential FITNESSGRAM technical reference manual (pp. 5772). Dallas, TX: The Cooper Institute for Aerobics Research.

Lohman, T. G. (1981). Skinfolds and body density and their relation to body fatness: A review. Human Biology, 53, 181-225.

Mann, H. B., \& Whitney, D. R. (1947). On a test of whether one of two random variables is stochastically larger than the other. Annals of Mathematical Statistics, $18,50-60$.

Meredith, M. D., \& Welk, G. J. (2007). Fitnessgram/ Activitygram Test Administration Manual. New York: Cooper Institute.

Murphy, N. A., \& Carbone, P. S. (2008). American Academy of Pediatrics Council on Children With Disabilities, Pediatrics, 121(5), 1057-61. 
National Center for Health Statistics. (2010). CDC growth charts: Percentile data files with LMS values. Retrieved from: http://www-users.med.cornell. $\mathrm{edu} / \sim$ spon/picu/calc/agecalc.htm.

Okely, A. D., Booth, M. L., \& Chey, T. (2004). Relationships between body composition and fundamental movement skills among children and adolescents. Research Quarterly for Exercise and Sport, 75(3), 238-247.

Pate, R. R., Ross, J. G., Baumgartner, T. A., \& Sparks, R. E. (1987). The modified pull-up test. Journal of Physical Education, Recreation, and Dance, 57(9), 71-73.

Patterson, P., Wiksten, D. L., Ray, L., Flanders, C., \& Sanphy, D. (1996). The validity and reliability of the back saver sit-and-reach test in middle school girls and boys. Research Quarterly for Exercise and Sport, 67, 448-451.

Pitetti, K. H., Beets, M. W., \& Combs, C. (2009). Physical activity levels of children with intellectual disabilities during school. Medicine and Science in Sports and Exercise, 41(8), 1580-1586.

Pitetti, K. H., \& Yarmer, D. A. (2002). Lower body strength of children and adolescents with and without mild mental retardation: A comparison. Adapted Physical Activity Quarterly, 19, 68-81.

Pitetti, K. H., Yarmer, D. A., \& Fernhall, B. (2001). Cardiovascular fitness and body composition in children and adolescents with and without mental retardation. Adapted Physical Activity Quarterly, 18, 124-141.

Plowman, S. A., \& Corbin, C. B., (1994). Muscular strength, endurance, and flexibility. In The Prudential FITNESSGRAM technical reference manual (pp. 7399). Dallas, TX: The Cooper Institute for Aerobics Research.

Pon, S. (2009). Medical calculators: Age calculator. Pediatric Critical Care Medicine, Cornell University. Retrieved from: http://www-users.med.cornell. $\mathrm{edu} / \sim \operatorname{spon} / \mathrm{picu} / \mathrm{calc} /$ agecalc.htm.

Robertson, L. D., \& Magnusdottir, H. (1987). Evaluation of criteria associated with abdominal fitness testing. Research Quarterly for Exercise and Sport, 58, 355-369.

Temple, V. A., Frey, G. C., \& Stanish H. I. (2006). Physical activity of adults with mental retardation: Review and research needs. American Journal of Health Promotion, 21(1), 2-12. 
Wilcoxon, F. (1945). Individual comparisons by ranking methods. Biometrics Bulletin, 1, 80-83.

Winnick, J. P. (2005a). Conceptual framework for the Brockport Physical Fitness Test. Adapted Physical Activity Quarterly, 22, 323-332.

Winnick, J. P. (2005b). Introduction to the Brockport Physical Fitness Test Manual. Adapted Physical Activity Quarterly, 22, 315-322.

Winnick, J. P., \& Short, F. X. (1999). The Brockport Physical Fitness Test manual. Champaign, IL: Human Kinetics.

Winnick, J. P., \& Short, F. X., (2005a). Test items and standards related to aerobic functioning on the Brockport Physical Fitness Test. Adapted Physical Activity Quarterly, 22, 333-355.

Winnick, J. P., \& Short, F. X., (2005b). Test items and standards related to body composition on the Brockport Physical Fitness Test. Adapted Physical Activity Quarterly, 22, 356-370.

Winnick, J. P., \& Short, F. X., (2005c). Test items and standards related to flexibility/range of motion on the Brockport Physical Fitness Test. Adapted Physical Activity Quarterly, 22, 401-417.

Winnick, J. P., \& Short, F. X., (2005d). Test items and standards related to muscle strength and endurance on the Brockport Physical Fitness Test. Adapted Physical Activity Quarterly, 22, 371-400.

Kathy DAVIS, Ph.D. is an Associate Professor of Physical Education Teacher Education in the Department of Exercise and Sport Science at East Carolina University, Greenville, North Carolina. She received a Ph.D. in Physical Education Curriculum and Instruction from Temple University in Philadelphia, Pennsylvania. She serves on the Board of Directors for the National Association for Sport and Physical Education (NASPE) and as the Chairperson of the Editorial Board for the Journal of Physical Education, Recreation, and Dance (AAHPERD). She also serves as the NASPE Publications Coordinator, and as a program reviewer for the National Council for the Accreditation of Teacher Education (NCATE). Her research interests involve diversity issues involving gender, race, and disability in relation to school physical education. E-mail address: daviska@ecu.edu 
Guili ZHANG, Ph.D. is an Assistant Professor of Research Methodology in the Department of Curriculum and Instruction, East Carolina University. She received a Ph.D. in Research and Evaluation Methodology from the University of Florida. She serves as Chair of the American Educational Research Association (AERA) Quantitative Dissertation Award Committee and Editor of Journal of Curriculum and Instruction. Dr. Zhang is a recipient of the Frontiers in Education Benjamin J. Dasher Best Paper Award, the American Society for Engineering Education Best Paper Award, and the Edward C. Pomeroy Award for Outstanding Contributions to Teacher Education from the American Association of Colleges for Teacher Education (AACTE). Her research interests involve applied quantitative research designs, categorical data analysis, longitudinal data management and analysis, large scale data analysis, program assessment and evaluation, meta-analysis, and mixedmethods research.

Patricia S. HODSON, Ph.D. is a Clinical Associate Professor (PT, DPT, PCS) and Director of Clinical Education in the Department of Physical Therapy, East Carolina University. Dr. Hodson received a BSPT from ECU and MS degree in Child Development and Family Relations from ECU. She completed the requirements for the Doctor of Physical Therapy in the distance education program at Simmons College in Boston. Dr. Hodson is a board certified pediatric specialist. Her clinical experience includes staff physical therapist positions at Beaufort County Hospital, Pitt County Memorial Hospital, and Greenville Developmental Evaluation Clinic. She served two terms on the North Carolina Board of Physical Therapy Examiners and is currently serving a third term. Dr. Hodson has published articles and abstracts in the Journal of Physical Therapy, Developmental Medicine and Child Neurology, Clinical Kinesiology, and Archives of Physical Medicine and Rehabilitation.

James T. DECKER, Ph.D. is an Associate Professor of Adapted Physical Education in the Department of Exercise and Sport Science, East Carolina University. He received a Ph.D. in Adapted Physical Education from The Ohio State University. He now serves as the Associate Dean of the College of Health and Human Performance at ECU. His primary research interests involve the development of physical activity programs for children with disabilities and the training of adapted physical educators.

Boni B. BOSWELL, Ph.D. is an Associate Professor of Adapted Physical Education in the Department of Exercise and Sport Science, East Carolina University. She received a Ph.D. in Adapted Physical Education from Texas Women's University. Her primary research interests involve the development of physical activity programs for children with disabilities and the training of adapted physical educators. 